\title{
RETRACTED ARTICLE: Thermophysical Properties of the Organic/Inorganic Nanocomposites Prepared with a Microwave Process
}

\author{
Sok Won Kim • Jaeran Lee • Seog Young Yoon • \\ Kwon Taek Lim
}

Received: 6 October 2008 / Accepted: 8 June 2009 / Published online: 23 June 2009 (C) Springer Science+Business Media, LLC 2015

At the request of the author, this paper is formally retracted as it duplicates a significant amount of material previously published in this article:

Key Engineering Materials, Vols. 317-318 (2006) pp. 669-672.

Preparation of Organic/Inorganic Nanocomposites with Microwave Process.

S. W. Kim $(\varangle)$ · J. Lee

Department of Physics, University of Ulsan, Ulsan 680-749, Republic of Korea e-mail: sokkim@ulsan.ac.kr

\section{S. Y. Yoon}

School of Material Science \& Engineering, Busan National University, Busan 609-735, Republic of Korea

K. T. Lim

Division of Image \& Information Engineering, Pukyung National University, Pusan 608-739, Republic of Korea 